Rev Chil Salud Pública 2012; Vol 16 (3): 208-209

Editorial invitada

\section{Chile: La buena salud de la investigación en salud}

\author{
CHILE: GOOD HEALTH IN HEALTH RESEARCH
}

En todos los sectores del país existe un amplio consenso respecto a que el desarrollo de la investigación científica en Chile pasa por un buen momento. El importante apoyo que otorga la Comisión Nacional de Investigación Científica y Tecnológica (Conicyt) en este ámbito, ha sido recientemente objetivado por su Presidente, en una carta enviada a la prestigiosa revista Science. . En esta se destaca el interés y compromiso con el desarrollo de la ciencia en el país, materializados en el incremento del presupuesto que la institución ha adjudicado en los últimos años.

\section{La inversión y apoyo a la investigación en salud en Conicyt}

Existe también acuerdo, tanto a nivel nacional como internacional, sobre la trascendencia de la investigación en el área de la salud. Conicyt cuenta con siete fondos a los que pueden postular investigadores del Área de las Ciencias Médicas y de la Salud, ampliamente difundidos y conocidos por los investigadores chilenos. Algunos de estos fondos disponen de diferentes instrumentos o concursos, cuyos montos adjudicados a proyectos de esta área en 2011, fueron los siguientes: Fondo Nacional de Investigación y Desarrollo en Salud (Fonis): \$956.829.000.-; Concurso Regular de Fondecyt, adjudicados a Medicina: \$10.076.681.000.-; Concurso Iniciación Fondecyt: \$ 1.336.368.000.-; Posdoctorados Fondecyt: \$ 536.200.000.-; y Concurso de Investigación y desarrollo de Fondeff: \$2.482.609.000.- En suma, a través de solo cinco de sus instrumentos, Conicyt otorgó apoyo a la investigación en salud en 2011, por un monto mayor a 15 mil millones de pesos (30 millones de dólares) ${ }^{* *}$.

La investigación en salud puede también acceder a apoyo en Conicyt a través de otros instrumentos, como la Investigación Asociativa o el Apoyo para Intercambios y Formación de Redes Internacionales de Investigación.

Desde el punto de vista del soporte a la generación de masa crítica de investigadores y profesionales de excelencia, la Comisión dispone también de fondos a los que pueden postular investigadores del Área de las Ciencias Médicas y de la Salud, como los programas de:

- Formación de Capital Humano Avanzado: becas para realizar estudios de magsteres y doctorados tanto en Chile como en el extranjero, así como apoyo a la Formación en sub-especialidades médicas en el extranjero.

- Inserción de Capital Humano Avanzado: fondos para la realización de tesis doctoral en el sector productivo y para la inserción de investigadores junior en el sector productivo y en la academia.

La importancia y el apoyo a la investigación en salud pública

Una mención en particular merece la investigación en el área de la salud pública, la importancia de respaldarla y el impacto significativo que esta genera.

El documento sobre investigación orientada a mejorar la salud de la Organización Mundial de la Salud (OMS), del año 2004, señala entre sus conclusiones respecto de este tipo de investigación, que: "... se debe gestionar con mayor efectividad si queremos que sirva para fortalecer los sistemas sanitarios
MARÍA XIMENA LUENGO(1)

(1)Directora Ejecutiva Fonis/ Minsal-Conicyt 
y la confianza de la población en la ciencia y en los científicos". Recalca también que: "hay que poner más énfasis en convertir el conocimiento en acción para mejorar la salud y llenar el vacío existente entre lo que se conoce y lo que realmente se está haciendo"*.

Así también, el Ministerio de Salud en Chile en su documento Estrategia Nacional de Salud 20112020, en el Objetivo 7 destinado a Fortalecer la institucionalidad del sector salud, determinó como estrategia: Fortalecer la investigación en salud ${ }^{* *}$.

Sin embargo, realizar este tipo de investigación no está exento de dificultades. Un reciente estudio de la Escuela de Salud Pública de la Universidad de Chile, que revisa el estado del arte de la investigación en salud pública en el país, señala que un $87 \%$ de las investigaciones en esta área fueron realizadas por instituciones académicas y que, entre las principales dificultades y desafíos percibidos por los investigadores, se mencionan los problemas relacionados con el financiamiento y con la formación de equipos humanos dedicados a la investigación ${ }^{\star * *}$.

En este contexto, el Fonis constituye uno de los principales instrumentos disponibles para apoyar la generación de nuevos conocimientos e investigaciones que contribuyan a mejorar la toma de decisiones en salud, tanto si nos referimos al diseño de políticas sanitarias como si nos situamos en el nivel más específico de las decisiones clínicas.

Entre sus objetivos considera también el apoyo a la formación de investigadores. A través de este Fondo, actualmente un importante número de profesionales e investigadores -a lo largo de todo el país-, está desarrollando avances destinados a impactar definitivamente nuestra calidad de vida actual y futura.

El interés que genera el concurso regular de Fonis, que consta de dos etapas, lo convierte en un fondo altamente competitivo. Grosso modo, se puede señalar que en promedio entre 2007 y 2011, una de cada cuatro propuestas presentadas fue seleccionada para continuar en concurso en la segunda etapa (24\%). En líneas generales, el porcentaje de adjudicación en los últimos tres años ha bordeado poco más del 10\%. En 2011, la adjudicación alcanzó al $13 \%$ de todas las propuestas presentadas, esto gracias al esfuerzo realizado en Conicyt por aumentar los fondos de apoyo, acorde con la alta demanda y en respuesta a que el nivel de las propuestas ha ido paulatinamente mejorando. El esfuerzo especial realizado los últimos años, ha incrementado los fondos adjudicados en aproximadamente 250 millones de pesos cada año; esto representa un incremento del $55 \%$ para 2010 y de un $35 \%$ al 2011, con relación al año anterior.

Fonis recibe propuestas de instituciones públicas o privadas sin fines de lucro, como servicios de salud, hospitales, universidades, centros de estudios, municipios, institutos profesionales y todos quienes se planteen el desarrollo de investigaciones científicas con aplicaciones clínicas y de salud pública. En 2011, se acordó incrementar el monto del presupuesto total al que pueden postular los proyectos, de 25 a 30 millones de pesos.

Desde el año 2004, Fonis ha asignado más de 3.200 millones de pesos a 178 proyectos de investigación en distintas áreas y, es importante destacar que uno de cada tres proyectos proviene de regiones distintas de la Metropolitana.

Este fondo financia proyectos realizados por personas que trabajan directamente en los temas que se plantean investigar, por lo que sus resultados constituyen un aporte efectivo y atingente para resolver problemas cotidianos del quehacer en salud, permitiendo la implementación de la evidencia científica, cuando corresponde, en un corto plazo.

Todos estos datos nos hablan de un modelo interesante de inversión en investigación aplicada en salud, de una iniciativa que ha podido financiar proyectos de excelencia en diversas áreas, los que demuestran un alto grado de compromiso e interés de los investigadores nacionales por contribuir con la salud pública en el país.

Como una iniciativa conjunta del sector salud y Conicyt, Fonis expresa claramente la voluntad de transferir los conocimientos generados a través de la investigación científica, al objetivo de alcanzar mejores niveles de salud y bienestar para la población en Chile.

\footnotetext{
* Organización Mundial de la Salud, OMS (2004). Informe Mundial sobre el conocimiento orientado a mejorar la salud. Fortalecimiento de los sistemas Sanitarios. Ginebra, Suiza.

** Ministerio de Salud, Gobierno de Chile (2011). Metas 2011-2020. Elige vivir sano. Estrategia nacional de salud para el cumplimiento de los Objetivos Sanitarios de la década 2011-2020

*** Solimano G., Alarcón A., Sepúlveda N (2011). Estado del Arte de la investigación en Salud Pública en Chile 1989 -2009. Informe de Resultados Programa Domeyko en Salud, Facultad de Medicina, Escuela de Salud Pública. Santiago de Chile.
} 